\title{
A clinico-epidemiological profile study of venereal genital dermatoses in a tertiary hospital in southern Nigeria: A 5-year review
}

\author{
Madubuko Cynthia Roli, ${ }^{1}$ Adejumo Oluseyi Ademola ${ }^{2}$ \\ ${ }^{1}$ Department of Medicine, University of Benin Teaching Hospital, Benin City; ${ }^{2}$ Department of Medicine, \\ University of Medical Sciences, Ondo State, Nigeria
}

\begin{abstract}
Venereal genital dermatoses are cutaneous morbidities contracted and transmitted by sexual contact. They are caused by microorganisms that survive on the skin or mucous membranes, or that are transmitted via semen, vaginal secretions, or blood during sexual intercourse. Data on pattern of presentation and diagnoses of venereal genial dermatoses are limited in Nigeria. The aim of this study was to determine the pattern of presentation of venereal genital dermatoses over a 5-year period in the University of Benin Teaching Hospital (UBTH), Benin City, Edo State, Nigeria. Information extracted from patients' medical records included socio-demographic data, duration of symptom, distribution of lesions, symptomatology and diagnoses of the venereal genital dermatoses. There were 121 patients with venereal genital dermatoses amongst 1600 new cases seen over the 5-year period constituting a
\end{abstract}

Correspondence: Madubuko Cynthia Roli, Department of Medicine, University of Benin Teaching Hospital, Benin City.

E-mail: rolimadubuko@yahoo.com

Key words: Venereal; dermatoses; genital; anogenital wart.

Conflict of interest: The authors declare no conflict of interest.

Availability of data and materials: All data generated or analyzed during this study are included in this published article.

Ethics approval and consent to participate: This study was approved by Health Research Ethics Committee of the University of Benin Teaching Hospital Benin city, Nigeria. The protocol number of the approval was $\mathrm{ADM} / \mathrm{E} / 22 / \mathrm{A} / \mathrm{VOL} . \mathrm{VII} / 14831014$. Approval was gotten from the ethical committee before obtaining data from the patients records for this study.

Informed consent: Written informed consent was obtained from a legally authorized representative(s) for anonymized patient information to be published in this article.

Received for publication: 14 May 2021.

Revision received: 10 June 2021.

Accepted for publication: 10 June 2021.

This work is licensed under a Creative Commons Attribution NonCommercial 4.0 License (CC BY-NC 4.0).

(C) Copyright: the Author(s), 2021

Licensee PAGEPress, Italy

Annals of Clinical and Biomedical Research 2021; 2:147

doi:10.4081/acbr.2021.147 prevalence of $7.6 \%$. The male:female ratio of the study population was 1.5:1 and their mean age was $26.2 \pm 10.5$ years. The predominant symptoms at presentation were abnormal genital growth in $108(89.3 \%)$, genital ulcer in $12(9.9 \%)$, and genital pain in 11 (9.1\%). The common venereal genital dermatoses were anogenital warts in $108(89.3 \%)$ and genital herpes in $11(9.1 \%)$. Venereal genital dermatoses are a relatively common dermatological presentation in UBTH and anogenital wart was the most common type. Venereal genital dermatoses were more common in the young age group and males. The most predominant site of affectation was the vulva in females and penis in males.

\section{Introduction}

Dermatoses involving genital areas are not always transmitted sexually. ${ }^{1}$ They can be divided into two groups: venereal and nonvenereal dermatoses. The diseases that are not sexually transmitted are referred as non-venereal dermatoses. Non-venereal genital dermatoses include a wide array of diseases with varied etiologies. ${ }^{2}$ Venereal genital dermatoses are cutaneous morbidities contracted and transmitted by sexual contact. They are caused by microorganisms that survive on the skin or mucous membranes, or that are transmitted via semen, vaginal secretions, or blood during sexual intercourse. The common venereal dermatoses include anogenital warts, genital herpes, syphilis, chancroid, donovanosis and lymphogranuloma venerum.

Data on pattern of presentation and diagnoses of venereal dermatoses are still limited especially in Nigeria probably because reporting of some sexually transmitted diseases like anogenital warts is not mandatory. ${ }^{3,4}$ The aim of this study was to describe the clinic-epidemiological pattern of venereal dermatoses observed over a 5-years period in the University of Benin Teaching Hospital, Benin City, Edo State, Nigeria.

\section{Materials and Methods}

This was a retrospective descriptive study that was carried out in the University of Benin Teaching Hospital, Benin City, Edo State. Medical records of all patients with venereal dermatoses who attended the out-patient clinic between December 2014 to December 2019 were analyzed. Information extracted from the case notes were socio-demographic information, clinical presentation and diagnosis of venereal dermatoses. The diagnosis of venereal dermatoses was essentially clinical, confirmed where expedient with a skin biopsy, swabs from ulcers for microscopy culture and sensitivity, dermoscopy, treponemal and non-treponemal tests where applicable for identifying syphilitic lesions. 


\section{Ethical considerations}

This study was approved by Health Research Ethics Committee of the University of Benin Teaching Hospital Benin city, Nigeria. The protocol number of the approval was ADM/E/22/A/VOL.VII/14831014.

\section{Data analysis}

Data entry and analysis was done using IBM Statistical Package for Social Science version 21. Categorical variables were expressed as frequencies and proportions. Continuous variables were expressed as mean \pm standard deviation. Data were displayed in tables and charts.

\section{Results}

The study was comprised of 121 clients with venereal genital dermatoses accounting for $7.8 \%$ of 1600 new patients attending dermatology outpatient clinic during the study period (Table 1). There were 73 men and 48 women (M:F 1.5:1) aged between 1 and 80 years. The mean age of the study participants was $26.2 \pm 10.5$ years. The clients were distributed across all age groups; 113 $(93.4 \%)$ were $\leq 40$ years, $6(5 \%)$ were $41-64$ years while $2(1.7 \%)$ were $\geq 65$ years. The duration of symptoms varied between 1 month and 8 years and the mean duration of symptoms was $11.1 \pm 13.2$ months.

The sites of the lesions were penis $59(48.8 \%)$, vulva 40 (33.1\%), anus $13(10.7 \%)$, scrotum $5(4.1 \%)$, vagina $3(2.5 \%)$ and gluteal region $1(0.8 \%)$ The clinical presentation were abnormal genital growth $108(89.3 \%)$, genital ulcer 12 (9.9\%), genital pain $11(9.1 \%)$, genital itching $4(3.3 \%)$, and genital rash $1(0.8 \%)$.

The venereal genital dermatoses observed in this study were anogenital warts $108(89.3 \%)$, genital herpes $11(9.1 \%)$, chancroid $1(0.8 \%)$ and molluscum contagiosum $1(0.8 \%$, Figure 1$)$.

The venereal genital dermatoses occurred more in males 73 $(60.3 \%)$ vs 48 (39.7\%) for females. This observation was not statistically significant ( $\mathrm{P}>0.05$, Figure 2 ). The young population had a higher prevalence of venereal genital dermatoses $113(93.4 \%)$ compared to the middle aged and elderly. This observation was statistically significant $\mathrm{p}<0.001$ Figure 3 .

\section{Discussion}

More than 1 million Sexually Transmitted Infections (STIs) are acquired every day worldwide. ${ }^{2}$

In this study, venereal genital dermatoses accounted for $7.8 \%$ of cases seen in the dermatology out -patient clinic. This is comparable to findings of Nnoruka et al. where they found sexually transmitted dermatoses to account for $5.4 \%$ of cases seen in their outpatient clinic. ${ }^{5}$ Anogenital warts was the most frequently diagnosed venereal genital dermatoses in our study and accounted for $89.3 \%$ of all venereal genital dermatoses. This is similar to previous report that showed that anogenital Human Papillomavirus (HPV) is the most frequent sexually transmitted viral infection in the world. ${ }^{6}$ It can result in cancers or benign skin and mucosal tumors. Anogenital wart commonly presents as visible lesions which may occur as single or multiple papules on the vulva, perineum, perianal area, vagina, cervix, penis, anus, scrotum and urethra (Figure 4). ${ }^{5}$ Majority of the lesions are self-limiting and often regress spontaneously within 6 months to 6 years of onset of symptoms. ${ }^{7}$ Approximately $90 \%$ of all genital warts are related to HPV types 6 and 11 which are least likely to have neoplastic potential. ${ }^{8}$

There was a male preponderance of anogenital warts in our study, although this was not statistically significant. Our finding was different from studies reported by Andahi et al. ${ }^{9}$ in North-eastern Nigeria, and Okesola et al. ${ }^{10}$ in South-western Nigeria where they reported a female preponderance in their study. This was explained by the increased incidence of warts in patients with venereal infections caused by Trichomonas vaginalis and Gardnerella spp which have been documented to be more common in females. ${ }^{9}$ The increased awareness about HPV vaccines for women may have begun to reflect in the incidence of HPV infections, thus reducing the prevalence rates amongst women. This may explain the male preponderance in our study. The advent of HPV vaccines and subsequent recommendation for use present an opportunity to lessen the burden of cervical disease among a population disproportionately affected.

In 2014, the Advisory Committee on Immunization Practices (ACIP) recommended routine HPV vaccination for the immunosuppressed women living with HIV (WLH) not more than age 26. ${ }^{11}$ Preferably, the HPV vaccine should be given prior to sexual initiation for the general population. ${ }^{12}$

The mean age of our clients with venereal genital dermatoses which was majorly anogenital wart was $26.2 \pm 10.5$ years. This is comparable with the finding of Saad et al. ${ }^{13}$ who reported that anogenital warts occurred more commonly in the 3rd decade of life in a study conducted in Northern Nigeria. This is also in keeping with report from some previous studies. ${ }^{14,15}$

Genital herpes was the second most common venereal genital dermatoses encountered in our study. It accounted for $9.1 \%$ of all venereal genital dermatoses in our study. Genital herpes is a common sexually transmitted viral disease. Higher prevalence rate of $40 \%$ was reported by Okwara et al. ${ }^{16}$ in a study done in this region over 10 years ago. The explanation for this disparity may be that in

Table 1. Clinico-epidemiologic data of study population.

\begin{tabular}{lc}
\hline Parameter & $\mathbb{N}=121 \mathrm{~N}(\%)$ \\
Age & \\
Mean \pm SD & $26.2 \pm 10.5$ years \\
Duration of symptoms & $11.1 \pm 13.2$ months \\
Mean \pm SD & \\
\hline Age group & $113(93.4)$ \\
Young (0-39years) & $6(5)$ \\
Middle aged (40-64years) & $2(1.7)$ \\
Elderly (>65) & \\
Sex & $73(60.3)$ \\
Male & $48(39.7)$ \\
Female & \\
Symptomatology & $1(0.8)$ \\
Rash & $12(9.9)$ \\
Ulcer & $108(89.3)$ \\
Abnormal growth & $4(3.3)$ \\
Pruritus & $11(9.1)$ \\
Pain & \\
Predominant anatomical site involved & \\
Penis & $59(48.8)$ \\
Vulva & $40(33.1)$ \\
Anus & $13(10.7)$ \\
Scrotum & $5(4.1)$ \\
Vagina & $3(2.5)$ \\
Gluteal region & $1(0.8)$ \\
\hline
\end{tabular}


our study diagnosis was mainly clinical and asymptomatic patients were not included in this study unlike their study where serology test was used for diagnosis.

Chancroid was seen in only one patient in this 5-years review period. Chancroid is an acute infection and auto-innoculable disease. ${ }^{17}$ The disease is more prevalent in Northern Nigeria partly due to permissive sexual practices especially for men. ${ }^{18}$ This hypothesis is in support of our finding of low prevalence observed in our center which is in the Southern part of Nigeria.

Molluscum contagiosum was seen in the genital area in $0.8 \%$ of patients observed with venereal dermatosis during the 5-years review. Molluscum contagiosum is a self-limited infectious dermatosis, which is frequent in the pediatric population, but rare in adults except for the sexually active adults, and immune-compromised individuals. It is transmitted mainly by direct contact with infected skin and clinically characterized by umbilicated pink or skin-colored papules. ${ }^{19,20}$ It is a frequent reason for consultation in pediatric dermatology and due to its self-limited nature, many of the lesions may resolve on their own without treatment within 12 18 months. This study was on venereal genital dermatoses explaining the paucity of the pediatric population.

The most predominant anatomical location of venereal genital

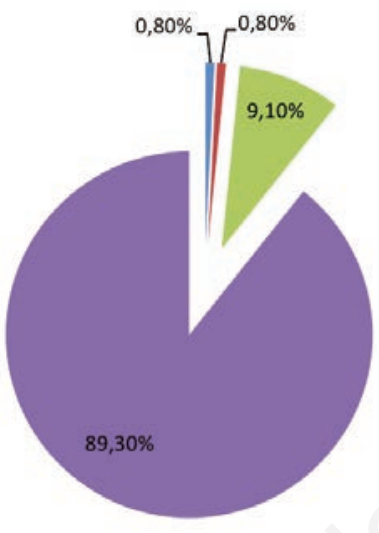

$=$ CHANCROID

- MOLLUSCUM

$=$ GENITAL HERPES

- ANOGENITAL WARTS

Figure 1. Frequency of venereal genital dermatoses.

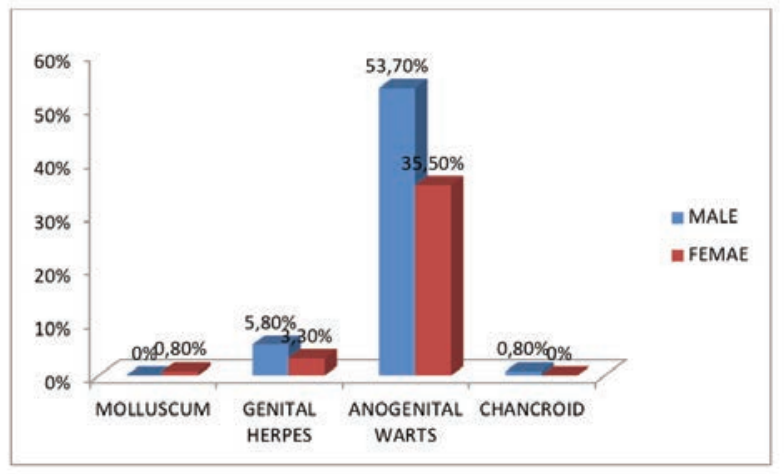

$P=0.526$

Figure 2. Gender distribution of venereal genital dermatoses. lesions in females in our study was the vulva (83.3\%). This finding was in keeping with report by Saad et al. ${ }^{13}$ in Northern Nigeria where $75 \%$ of the females in that study had their lesions on the vulva. ${ }^{13}$ Also, the penis $(80.8 \%)$ was the most common site of venereal genital lesions in males. This observation in our study was at variance to reports by Saad et al. ${ }^{13}$ where perianal area was observed to be the most predominant site for genital lesions among males. Homosexual practices have been associated with increased prevalence of anal warts. This difference may be related to the type of sexual practices such as anal sex among the male population studied, although this was not included as part of information extracted for our study.

The limitation of this study was that the findings may not reflect the characteristics of venereal genital dermatoses in a general population because it was hospital based. Also, because it was a retrospective study, the information that could be retrieved were limited by previous documentation.

In conclusion, venereal genital dermatoses is a relatively common dermatological presentation in the out-patient clinic and anogenital wart was the most common. It was more common in the young age group and males. The most predominant site of affectation were the vulva in females and penis in males.

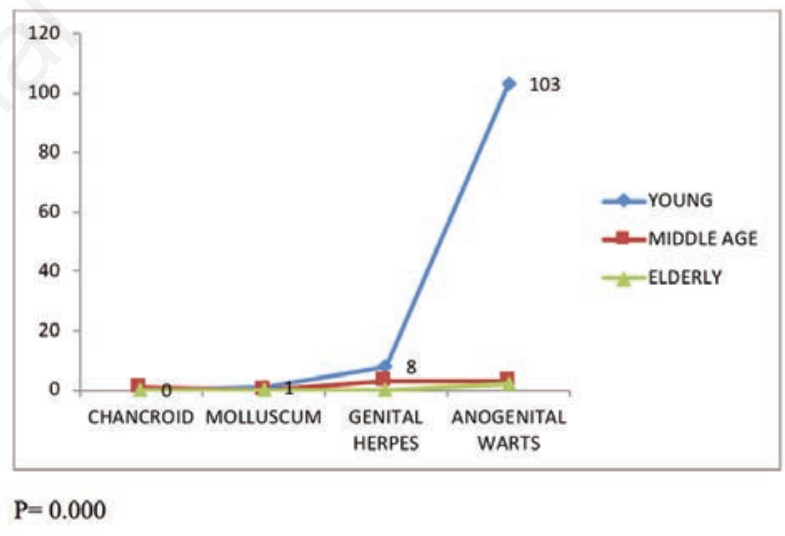

Figure 3. Age distribution of venereal genital dermatoses.

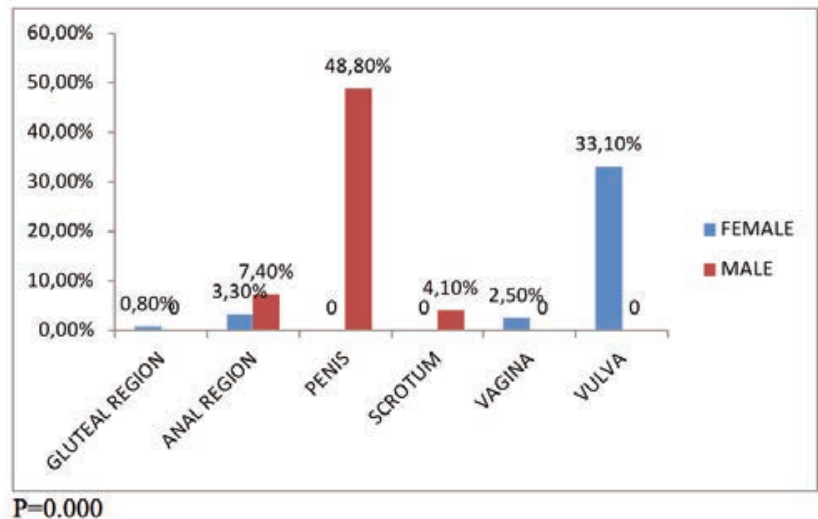

Figure 4. Pattern of distribution of venereal genital dermatoses. 
Ibadan. West Afr J Med 2000;19:195-9.

11. Markowitz LE, Dunne EF, Saraiya M, et al. Human papillomavirus vaccination: recommendations of the Advisory Committee on Immunization Practices (ACIP) MMWR Recommendations and reports: Morbidity and mortality weekly report Recommendations and reports/Centers for Disease Control 2014;63:1-30.

12. Kahn JA, Burk RD, Squires KE, et al. Prevalence and risk factors for HPV in HIV-positive young women receiving their first HPV vaccination. J Acquired Imm Def Syndr 2012;61:390-9.

13. Saad A, Mohammed S, Murtala A, Suleiman E. Anogenital warts in Northern Nigeria: A 10 year review. Niger Med J 2013;55:313-5.

14. Schiffman M, Castle PE. Human papillomavirus: Epidemiology and public health. Arch Pathol Lab Med 2003;127:930-4.

15. DiClemente RJ, Sales JM, Danner F, Crosby RA. Association between sexually transmitted diseases and young adults' selfreported abstinence. Pediatrics 2011;127:208-13.

16. Okwara B, Eze E, Onunu A, et al. Prevalence of HSV type 11 antibodies in HIV infected patients in Benin city Nigeria. Int J Biomed Res 2017;47-8

17. King A, Nicol C. Venereal Diseases. 3rd Edition. London: White Friars Press Ltd.; 1975.

18. Mbata TI, Onile BA, Agbonlahor DE, et al. Diagnosis and management of chancroid in Nigeria. Malawi Med J 2004;16:19-21.

19. Leung AKC, Barankin B, Hon KLE. Molluscumcontagiosum: an update. Recent Pat Inflamm Allergy Drug Discov 2017;11:22-31.

20. Gerlero P, Hernandez-Martin A. Update on the treatment of molluscum contagiosum in children. Actas Dermosifiliogr 2018;109:408-15. 